\title{
The role of non- $\beta$-lactam antimicrobials and screening for vancomycin resistance in methicillin resistant Staphylococcus aureus
}

\author{
Khan, F.*, Shukla, I. and Rivzi, M. \\ Department of Microbiology, JNMC Hospital, AMU, Aligarh, India, 202002. \\ E-mail:fatimasalmanshah@ymail.com \\ Received 13 June 2010; received in revised form 9 September 2010; accepted 9 September 2010
}

\begin{abstract}
The study was carried out to determine the prevalence of MRSA, VRSA and their current antimicrobial susceptibility pattern to various non- $\beta$-lactam antimicrobial agents to record the current status of MRSA response to commonly used antistaphylococcal antibiotics in Aligarh, India for a period of two years. A total of 430 Staphylococcus aureus strains were isolated from various clinical samples. Two hundred ninety $(67.44 \%)$ of $S$. aureus were isolated from pus and most of these were from orthopaedics $124(28.85 \%)$ and surgery wards 99 (23.02\%). One hundred thirty eight $(32.09 \%)$ strains were confirmed to be methicillin resistant by both phenotypic and genotypic methods. More than $80 \%$ of MRSA strains were multidrug resistant. However, all were uniformly sensitive to vancomycin and linezolid. Levofloxacin was the drug found to be resistant in just $16.47 \%$ MRSA strains. Vancomycin is the drug of choice for MRSA treatment. However, regular screening should be done for vancomycin intermediate and vancomycin resistant strains of Staphylococcus aureus. We suggest the use of levofloxacin for MRSA treatment since overuse of vancomycin can lead to the development of vancomycin resistance and more importantly it is beyond the scope of poor patients in developing countries like India.
\end{abstract}

Keywords: S. aureus, MRSA, VRSA, screening, non- $\beta$-lactam, multidrug resistance

\section{INTRODUCTION}

Staphylococcus aureus is an important human pathogen causing pyogenic, disseminated and toxin mediated infections (Oliveira et al., 2001; Enright et al., 2002; Robinson et al., 2003). The spectrum of $S$. aureus infections includes food poisoning, meningitis (Betley et al., 1992; Ish-Horowicz, 1992; Collee et al., 2006), toxic shock syndrome (Betley et al., 1992; Collee et al., 2006), as well as dermatological disorders ranging from minor infections and eczema to blisters and scalded skin syndrome (Winn et al., 2006). Infections caused by $S$. aureus used to respond to $\beta$-lactam and related group of antibiotics. However due to development of methicillin resistance amongst $S$. aureus isolates (MRSA); treatment of these infections has become problematic. MRSA is a serious threat to the hospitalised patients globally and it now represents a challenge for public health as community associated infections appear to be on the increase in both adults and children (Layton et al., 1995). Currently treatment options for MRSA infections are limited to very few and expensive drugs like teicoplanin and vancomycin. Already resistant to multiple classes of antibiotics, MRSA had been reported to acquire resistance even to vancomycin (Asadullah et al., 2003), so in the near future the treatment options for MRSA infections are going to shrink further. The development of resistance to multiple antibiotics and control of disease transmission by MRSA isolates in hospitals or communities have been recognised as a major challenge as the bacterial population that

${ }^{{ }^{*} \text { Corresponding author }}$ expresses the resistance varies according to the environmental conditions (Qureshi et al., 2004). Therefore the knowledge of prevalence of MRSA and VRSA and their antimicrobial profile become necessary in the selection of appropriate empirical therapy of these infections. We determined the prevalence of MRSA, VRSA and their current antimicrobial susceptibility pattern to various non- $\beta$-lactam antimicrobial agents to record the current status of MRSA response to commonly used antistaphylococcal antibiotics in western UP, India.

\section{MATERIALS AND METHODS}

The present study was conducted in the department of Microbiology, J.N. Medical College and Hospital, AMU, Aligarh during the period from August 2005 to July 2007. Various clinical specimens such as pus, blood, urine, CSF, pleural fluid, peritoneal fluid, ascitic fluid, bile, cervical swab, semen, conjunctival swabs and ear swabs received in the Microbiology laboratory were studied. The samples were cultured on $5-10 \%$ sheep blood agar, MacConkey agar, Mannitol salt agar and Robertson's cooked meat broth. Only those specimens from which staphylococci were isolated were included in the study. All the isolates suggestive of $S$. aureus were identified by the standard biochemical procedures (Collee et al, 2006). The methicillin susceptible strain ATCC 25923 was used as a control for the diagnostic procedures. All isolates were maintained in $0.5 \%-1 \%$ semisolid nutrient agar stabs and 
seeded with cork stoppers soaked with hot sterile paraffin until analysed (Colle and Miles, 1989).

\section{Oxacillin disc diffusion test}

All the isolates were subjected to oxacillin disc diffusion test using oxacillin $1 \mu \mathrm{g}$ disc. A $0.5 \mathrm{McF}$ arland turbidity standard suspension of the isolate was made and lawn culture was done on Mueller-Hinton agar (MHA) plates containing $4 \% \mathrm{NaCl}$. Plates were incubated at $37{ }^{\circ} \mathrm{C}$ for $18 \mathrm{~h}$ and zone diameters were measured. An inhibition zone diameter of $\leq 10 \mathrm{~mm}$ was reported as methicillin resistant and $\geq 13 \mathrm{~mm}$ was taken as methicillin sensitive.

\section{MIC determination of oxacillin}

MIC was determined by agar dilution test. 10 different dilutions of oxacillin were selected such that the concentrations that allowed determination of MIC breakpoints defining susceptible $(\leq 2 \mu \mathrm{g} / \mathrm{mL})(\mathrm{CLSI}, 2004)$ and resistant ( $\geq 4 \mu \mathrm{g} / \mathrm{mL}$ ) (CLSI, 2004) values were included. Lowest concentration at which the growth was inhibited by $80 \%$ or more was recorded as MIC.

\section{PCR amplification for mec $A$ and fem $B$ genes}

Multiplex PCR (Geha et al., 1994) was carried out on all the $S$. aureus strains found methicillin resistant on MIC determination. All the MRSA strains were for the mec A and femB genes using the following oligonucleotides sequence.mec A1-5' GTA GAA ATG ACT GAA CGT CCG ATA A-3', mec A2-5' CCA ATT CCA CAT TGT TTC CGT CTA A-3', fem B1-5' TTA CAG AGT TAA CTG TTA CC-3', fem B2-5' ATA CAA ATC CAG CAC GCT CT-3'. A $50 \mu \mathrm{L}$ $\mathrm{PCR}$ reaction mixture consisted of $45 \mu \mathrm{L}$ of mastermix containing PCR buffer (1X), d NTP mix (0.2 mM of each), primer $(0.5 \mu \mathrm{M})$, Taq DNA polymerase $(0.25 \mathrm{U})$, and $\mathrm{MgCl}_{2}(1.5 \mathrm{mM})$ with $5 \mu \mathrm{L}$ of template DNA. Cycling parameters of PCR were set as follows: hot start $94^{\circ} \mathrm{C}$ for 4 min followed by 35 cycles of melting at $94^{\circ} \mathrm{C}$ for $45 \mathrm{~s}$, annealing at $50{ }^{\circ} \mathrm{C}$ for $45 \mathrm{~s}$, and extension at $72{ }^{\circ} \mathrm{C}$ for 1 min. Analysis of amplified products was done by gel electrophoresis. Amplicons of $310 \mathrm{bp}$ were consistent with mec $A$ and of 651 bp with fem $B$ gene amplification.

\section{Screening of vancomycin resistance}

\section{Himartshu Method}

Overnight grown cultures were adjusted to $0.5 \mathrm{McF}$ arland approximately $1.5 \times 10^{8} \mathrm{cfu} / \mathrm{mL}$ and $10 \mu \mathrm{L}$ spots was inoculated on $\mathrm{BHI}$ agar containing $4 \mu \mathrm{g} / \mathrm{mL}$ of vancomycin. The plates were incubated at $35{ }^{\circ} \mathrm{C}$ for $24-48 \mathrm{~h}$. Appearance of any growth indicated vancomycin resistance (Wootton et al., 2001).

\section{CDC Method}

Overnight grown cultures were adjusted to $0.5 \mathrm{Mc}$-Farland approximately $1.5 \times 10^{8} \mathrm{cfu} / \mathrm{mL}$ and diluted 100 times to get an inoculum of approximately $1.5 \times 10^{6} \mathrm{cfu} / \mathrm{mL}$. Ten $\mu \mathrm{L}$ spots of the cultures were inoculated on $\mathrm{BHI}$ agar containing $6 \mu \mathrm{g} / \mathrm{mL}$ of vancomycin. The plates were incubated at $35^{\circ} \mathrm{C}$ for $24-48 \mathrm{~h}$. Appearance of any growth indicated vancomycin resistance (Tenover et al., 2001).

\section{Method by Tenover et al. (2001)}

Overnight grown cultures were adjusted to $0.5 \mathrm{Mc}$-Farland $1.5 \times 10^{8} \mathrm{cfu} / \mathrm{mL}$ and diluted 100 times to get an inoculum of approximately $1.5 \times 10^{6}$. Ten $\mu \mathrm{L}$ of both the above mentioned inoculums were spot inoculated on MHA agar containing $5 \mu \mathrm{g} / \mathrm{mL}$ of vancomycin. The plates were incubated at $35^{\circ} \mathrm{C}$ for $24-48 \mathrm{~h}$. Appearance of any growth indicated vancomycin resistance (Huber et al., 1999).

\section{MIC determination of vancomycin}

Vancomycin MICs for the clinical isolates were determined by agar dilution method in Mueller Hinton agar according to the protocol of National committee for Clinical Laboratory Standards (NCCLS). Eight different dilutions of van-comycin were selected such that the concentrations that allowed determination of MIC breakpoints defining susceptible ( $\leq 4 \mu \mathrm{g} / \mathrm{mL}$ ) (Tenover et al., 2001), intermediate $(8-16 \mu \mathrm{g} / \mathrm{mL}$ ) (Tenover et al., 2001) and resistant $(\geq 32 \mu \mathrm{g} / \mathrm{mL}$ ) (Tenover et al., 2001) values were included. Lowest concentration at which the growth was inhibited by $80 \%$ or more was recorded as MIC.

Antibiotic susceptibility testing of all the MRSA strains was done using Kirby Bauer's disk diffusion method (CLSI, 2000) for the following non- $\beta$-lactam antimicrobial agents: amikacin $30 \mu \mathrm{g}$, ciprofloxacin $5 \mu \mathrm{g}$, clindamycin 2 $\mu \mathrm{g}$, cotrimoxazole $25 \mu \mathrm{g}$, erythromycin $15 \mu \mathrm{g}$, gatifloxacin $5 \mu \mathrm{g}$, gentamycin $10 \mu \mathrm{g}$, levofloxacin $5 \mu \mathrm{g}$, linezolid $30 \mu \mathrm{g}$, ofloxacin $5 \mu \mathrm{g}$, sparfloxacin $5 \mu \mathrm{g}$, tobramycin $10 \mu \mathrm{g}$, vancomycin $30 \mu \mathrm{g}$.

\section{RESULTS}

A total of $430 S$. aureus were isolated from various clinical specimens. The majority of $S$. aureus strains were isolated from pus $290(67.44 \%)$ followed by urine 62 $(14.42 \%)$ and blood $49(11.39 \%)$ (Table 1) and around $50 \%$ of these were from the orthopaedics 124 (28.85\%) and the surgery wards $99(23.02 \%)$ (Table 2). Out of 430 S. aureus strains $143(33.25 \%)$ were found to be methicillin resistant on phenotypic detection by oxacillin disc diffusion test. However, on genotypic detection with the help of multiplex PCR 138 (32.09\%) strains had both mec A (310 bp) and fem B (651 bp) and were confirmed to be methicillin resistant. MIC of all the MRSA isolates was more than $4 \mu \mathrm{g} / \mathrm{mL}$ but none was greater than 256 $\mu \mathrm{g} / \mathrm{mL}$. Prevalence of MRSA was highest amongst the orthopaedics 64 (46.38\%) and the surgery 49 (35.50\%) wards (Table 2). Out of 138 MRSA strains $112(81.16 \%)$ were MDR-MRSA (resistant to > 3 non- $\beta$-lactam antibiotics belonging to different groups) and only 26 (18.84\%) strains were non-MDR-MRSA (Table 3). Among the multidrug-resistant isolates maximum 21 (15.22\%) 
Table 1: Isolation rate of $S$. aureus in relation to specimen $(n=430)$

\begin{tabular}{lc}
\hline Specimen & No. of isolates (\%) \\
\hline Pus (P) & $290(67.44)$ \\
Blood (B) & $49(11.39)$ \\
Endocervical swab (Cx) & $1(0.23)$ \\
Ear swab (E) & $2(0.47)$ \\
Urine (U) & $62(14.42)$ \\
CSF (CSF) & $7(1.63)$ \\
Conjunctival swab (Cj) & $2(0.47)$ \\
Drain tip (Dt) & $1(0.23)$ \\
Foley's tip (Ft) & $2(0.47)$ \\
Fluids (Pleural, ascitic, peritoneal) & $0(0.00)$ \\
Sputum and throat swab & $1(0.23)$ \\
Seminal fluid & $2(0.47)$ \\
Catheter tip & $11(2.5)$ \\
Umblical tip & $1(0.23)$ \\
\hline Total & $\mathbf{2 6 2 ( 1 0 0 )}$ \\
\hline
\end{tabular}

Table 2: Isolation rate of $S$. aureus from various wards in hospital

\begin{tabular}{lccc}
\hline Hospital wards & Non- MRSA & MRSA & $\begin{array}{c}\text { S. } \\
\text { aureus }\end{array}$ \\
\cline { 2 - 4 } & $\mathbf{N}(\%)$ & $\mathbf{N}(\%)$ & $\mathbf{N}$ \\
\hline Orthopaedics & $66(53.23)$ & $58(46.77)$ & 124 \\
Surgery & $64(64.65)$ & $35(33.35)$ & 99 \\
Paediatrics & $27(72.97)$ & $10(27.03)$ & 37 \\
Gynaecology & $29(75.32)$ & $9(23.68)$ & 38 \\
Medicine & $15(75.00)$ & $5(25.00)$ & 20 \\
ICU & $6(54.55)$ & $4(45.45)$ & 11 \\
ENT & $12(70.59)$ & $5(29.41)$ & 17 \\
TB and Chest & $10(100.00)$ & $0(0.00)$ & 10 \\
Ophthalmology & $9(90.00)$ & $1(10.00)$ & 10 \\
Skin & $20(95.24)$ & $1(4.76)$ & 21 \\
Plastic surgery & $15(65.22)$ & $8(34.78)$ & 23 \\
(Burn) & $19(95)$ & $1(5)$ & 20 \\
Nursery & $\mathbf{2 9 2 ( 6 7 . 9 1 )}$ & $\mathbf{1 3 8 ( 3 2 . 0 9 )}$ & $\mathbf{4 3 0}$ \\
\hline Total
\end{tabular}

Table 3: Pattern of resistance of MRSA isolates to other drugs $(n=138)$

\begin{tabular}{|c|c|c|}
\hline \multicolumn{2}{|c|}{ No. of other drugs } & \multirow{2}{*}{$\begin{array}{c}\text { No. of MRSA isolates (\%) } \\
0(0.00)\end{array}$} \\
\hline \multirow{4}{*}{ 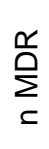 } & 0 & \\
\hline & 1 & $6(4.35)$ \\
\hline & 2 & $8(5.80)$ \\
\hline & 3 & $12(8.69)$ \\
\hline \multirow{9}{*}{ 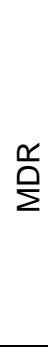 } & 4 & $9(6.52)$ \\
\hline & 5 & 21 (15.22) \\
\hline & 6 & $16(11.59)$ \\
\hline & 7 & $9(6.52)$ \\
\hline & 8 & $20(14.49)$ \\
\hline & 9 & 17 (12.32) \\
\hline & 10 & $14(10.14)$ \\
\hline & 11 & $6(3.62)$ \\
\hline & 12 & $0(0.00)$ \\
\hline & & $138(100.00)$ \\
\hline
\end{tabular}

were resistant to five antibiotics. Maximum resistance was shown to cotrimoxazole $(89.41 \%)$ followed by clindamycin $(83.53 \%)$. Among the quinolones ciprofloxacin was found to be at the bottom of the list with maximum resistance of $81.17 \%$, followed by gatifloxacin $(45.88 \%)$, sparfloxacin (38.88\%), and ofloxacin (23.53\%). Levofloxacin was found to be the best amongst the flouroquinolones with just $16.47 \%$ resistance. Amikacin was better at $60.00 \%$ resistance among the aminoglycosides with gentamicin showing resistance to $69.41 \%$ and tobramicin to $71.00 \%$ strains. Linezolid and vancomycin were the only two drugs to which all the strains of MRSA were uniformly sensitive. On screening for vancomycin resistance it was found that $11(7.97 \%)$ strains of MRSA were resistant to vancomycin by the Himartshu method, and $8(5.7 \%)$ by the method by Tenover and co-workers. However CDC method detected no vancomycin intermediate (VISA) or vancomycin resistant (VRSA) strains of $S$. aureus which was in correlation with MIC of vancomycin by the agar dilution method. The MIC for vancomycin of all the MRSA strains was found to be less than $4 \mu \mathrm{g} / \mathrm{mL}$.

\section{DISCUSSION}

MRSA is a major pathogen causing significant mortality and morbidity in the hospitals as well as among the communities. Many of the MRSA are becoming multidrug resistant and are susceptible only to glycopeptides antibiotics such as vancomycin (Mehta et al., 1998). Low level resistance even to vancomycin is emerging at present (Asadullah et al., 2003). The prolonged hospital stay, indiscriminate use of antibiotics, receipt of antibiotics before coming to the hospital etc. are the possible predisposing factors of MRSA emergence (Anupurba et al., 2003). The prevalence of MRSA in our hospital was found to be $32.44 \%$. Similar rate of MRSA isolation had also been reported from other parts of India (Anbumani et al., 2006; Dar et al., 2006; Gopalakrishnan et al., 2006; Oberoi and Varghese, 2006). However, a higher rate (of around $50 \%$ ) of MRSA prevalence is reported in some studies (Anupurba et al., 2003; Vidhani, 2001; Krishnan, 2002). The high prevalence of MRSA in these studies could be due to several factors like indiscriminate use of antibiotics and unethical treatment before coming to the hospital.

In the present study maximum isolates of $S$. aureus were from pus $(77.87 \%)$. This justifies the fact that $S$. aureus is a known cause of wound infections. Rate of MRSA isolation was also highest among the pus samples especially from the orthopaedics, surgery wards and the ICU. This might be because the patients in these wards are usually with open wounds and are debilitated. They undergo multiple interventions in the hospital which further increases the risk of MRSA infection due to multiple people involved as well as prolonged stay in the wards. Along with these factors, the patient is usually on multiple antibiotics. On antimicrobial sensitivity testing it was found the MRSA isolates were resistant to multiple groups of antibiotics. Around $80 \%$ of the strains were resistant to more than three non- $\beta$-lactam antibiotics and were 
multidrug resistant. However all the strains were uniformly sensitive to linezolid and vancomycin. Such an incidence of multidrug resistance had also been reported in other studies from North India (Vidhani, 2001; Dar et al., 2006). Among the flouroquinolones ciprofloxacin was reported to have highest resistance of $81.17 \%$. Similar to our findings, a high resistance of $90-98 \%$ had been reported for ciprofloxacin in other studies (Pulimood et al., 1996; Qureshi et al., 2004). Among the aminoglycosides gentamicin had been reported to be resistant in $63.6 \%$ isolates by Rajaduraipandi and co-workers (2006), similar to our findings. However some authors had quoted up to $97.8 \%$ resistance to gentamicin which is higher compared to ours (Qureshi et al., 2004). Linezolid and vancomycin were the only drugs to which all the strains were sensitive. However some authors had reported emerging vancomycin resistance with strains showing intermediate [VRSA] and complete resistance to vancomycin [VISA] (Asadullah et al., 2003; Gopalakrishnan, 2006). At present the proportion of MRSA with reduced susceptibility [VISA] to vancomycin is well known. Vancomycin resistance can be difficult to detect in clinical microbiology laboratories since sensitivity testing by disc diffusion method may sometimes misclassify intermediate susceptible isolates as fully susceptible. MIC determination by agar and broth dilution methods is considered the gold standard (Srinivasan et al., 2002) but it is beyond the scope of most of the micro-biology laboratories especially in the developing countries like India. The screening methods can be used for early and accurate detection of vancomycin resistance. Among the three screening methods evaluated in our study CDC method was found to be the most accurate with maximum correlation with the MIC of vancomycin detected by agar dilution method. Himartshu method and the method by Tenover and coworkers (2001) were found to be false positive in 11 $(7.97 \%)$ and $8(5.7 \%)$ isolates respectively. We recommend the use of the CDC method for regular screening for vancomycin resistance in all the microbiology laboratories. However, confirmation of vancomycin resistance should be done by the determination of MIC in all the isolates suspected to have VISA or VRSA. But overuse of vancomycin can promote the emergence of its resistance and more importantly in developing countries like India poor patients cannot afford treatment with these costly drugs. Another limiting factor for vancomycin use is the high rate of adverse affects caused by the same. We suggest that no antimicrobial should be used on regular basis for empirical treatment because of the rapidly changing pattern of drug resistance. So we recommend that proper screening of antimicrobial sensitivity pattern of Staphylococcus species should be done within each hospital on annual basis and empirical antimicrobial therapy should be modified accordingly. The next respon-sibility goes to the clinicians who should exercise caution in their use of vancomycin or any antimicrobial and help to prevent the spread of drug resistance by the rationale and proper use of antimicrobials.

\section{REFERENCES}

Anbumani, N., Wilson, A. A., Kalyani, J. and Mallika, M. (2006). Prevalence of methicillin resistant Staphylococcus aureus in a tertiary referral hospital in Chennai, South India. Indian Journal for Practicing Doctors 3, 2006-2009.

Anupurba, S., Sen, M. R., Nath, G., Sharma, B. M., Gulati, A. K. and Mohapatra, T. M. (2003). Prevalence of methicillin resistant Staphylococcus aureus in a tertiary referral hospital in Eastern Uttar Pradesh. Indian Journal of Medical Microbiology 21, 49-51.

Asadullah, S., Kakru, D. K., Thokar, M .A., Bhat, F. A., Hussain, N. and Shah, A. (2003). Emergence of low level vancomycin resisitance in MRSA. Indian Journal of Medical Microbiology 21, 196-198.

Baird, D. (2006). Staphylococcus: Cluster-forming GramPositive cocci. In: Mackey and McCartney practical Medical Microbiology. Collee, J. G., Fraser, A. G., Marmion, B. P. and Simmons, A. (eds.). Reed Elsevier India, NewDelhi, India. pp. 245-261.

Betley, M. J., Borst, D. W. and Regessa, L. B. (1992). Staphylococcal enterotoxins, toxic shock syndrome toxin and staphylococcal pyogenic exotoxins: a comparative study of their molecular biology. In: Biological Significance of Superantigens. Fleisher B., Karger, B. (eds.). Novapublishers New York. pp 1-35.

Clinical and Laboratory Standards Institute (2000). Wayne Pa: Methods for dilution antimicrobial suscseptibility tests for bacteria that grow aerobically. $5^{\text {th }}$ edn. Approved standard M7-A5.

Clinical and Laboratory Standards Institute (2004). Wayne Pa: Performance standards for antimicrobial susceptibility testing, $14^{\text {th }}$ information supplement. M100-S14.

Collee, J. G., Miles, R. S., Watt, W. (2006). Tests for identification of bacteria. In: Mackey and McCartney practical Medical Microbiology. Collee, J. G., Fraser, A. G., Marmion, B. P. and Simmons, A. (eds.). Reed Elsevier India, New Delhi, India. pp. 131-150.

Dar, J. A., Thoker, M. A., Khan, J. A., Ali, A., Khan, M. A., Rizwan, M., Bhat, K. H., Dar, M.J., Ahmed, N. and Ahmad, S. (2006). Molecular epidemiology of clinical and carrier strains of Methicillin Resistant Staphylococcus aureus (MRSA) in the hospital settings of North India. Annals of Clinical Microbiology and Antimicrobials 5, 22.

Enright, M. C., Robinson, D. A., Randle, G., Feil, E. J., Grundmann, H., Spratt and B. G. (2002). The evolutionary history of methicillin-resistant Staphylococcus aureus (MRSA). Proceedings of the National Academy of Sciences USA 99, 7687-7692.

Geha, J., Uhl, J. R., Gustaferro, C. A. and Persing,D. H. (1994). Multiplex PCR for identification of methicillin resistant staphylococci in the clinical laboratory. Journal of Clinical Microbiology 32, 1768-1772.

Gopalakrishnan, B. K., Saritha, K. M. and Hussain, A. (2006). Nosocomial methicillin resistant Staphylococcus aureus with reduced susceptibility to 
vancomycin. Indian Journal of Pathology and Microbiology 49, 311-312.

Huber, S. K., Mohammad, J. M., Fridkinn, S. K., Gaynes, R. P., McGowan, J. E., Tenover, F. C. (1999). Glycopeptide intermediate Staphylococcus aureus: Evaluation of novel screening methods and results of a survey of selected US hospitals. Journal of Clinical Microbiology 37, 3590-3593.

Ish-Horowicz, M. R., Melntyre, P. and Nade, S. (1992). Bone and joint infections caused by multiply resistant Staphylococcus aureus in a neonatal intensive care unit. Pediatrics Infectious Disease Journal 11, 82-87.

Krishnan, P. V., Miles, K. and Shetty, N. (2002). Detection of methicillin and mupirocin resistance in Staphylococcus aureus isolates using conventional and molecular methods: A descriptive study from a burn unit with high prevalence of MRSA. Journal of Clinical Pathology 55, 745-748.

Layton, M. C., Hierholzer, W. J. and Patterson, J. E. (1995). The evolving epidemiology of methicillin resisitant Staphylococcus aureus at a university hospital. Infection Control and Hospital Epidemiology 16, 12-17.

Mehta, A. P., Rodrigues, C., Sheth, K., Jani, S., Hakimiyan, A. and Fazalbhoy, N. (1998). Control of methicillin resistant Staphylococcus aureus in a tertiary care centre- A five year study. Journal of Medical Microbiology 16, 31-34.

Oberoi, A. and Verghese, S. R. (2006). A study of MRSA- a nosocomial pathogen in a tertiary care centre in Punjab. Indian Journal of Pathology and Microbiology 49, 313-314.

Oliveira, D., Thomasz, A. and deLencastre, H. (2001). The evolution of pandemic clones of methicillin resistant Staphylococcus aureus: Identification of two ancestral genetic backgrounds and the associated mec elements. Microbial Drug Resistance 7, 49-61.

Pulimood, T. B., Lalitha, M. K. and Jesudson, M. V. (1996). The spectrum of antimicrobial resistance among methicillin resisitant Staphylococcus aureus (MRSA) in a tertiary care hospital in India. Indian Journal of Medical Microbiology 103, 212-215.

Qureshi, A. H., Rafi, S., Qureshi, S. M. and Ali, A. M. (2004). The current susceptibility patterns of methicillin resistant Staphylococcus aureus to conventional antistaphylococcus antimicrobials at Rawalpandi. Pakistan Journal of Medical Sciences 20, 361-364.

Rajaduraipandi, K., Mani, K. R., Panneerselvam, K., Mani, M., Bhaskar, M. and Manikandan, P. (2006). Prevalence and antimicrobial susceptibility of methicillin resistant Staphylococcus aureus: A multicentre study. Indian Journal of Medical Microbiology 24, 34-38.

Robinson, D. A. and Enright, M. C. (2003). Evolutionary models of the emergence of methicillin-resistant Staphylococcus aureus. Antimicrobial Agents Chemotherapy 47, 3926-3934.
Srinivasan, A., Dick, J. D. and Trish, M. P. (2002). Vancomycin resistance in Staphylococci. Clinical Microbiology Review 15, 430-438.

Tenover, F. C., Biddle, J. W. and Lancaster, M. V. (2001). Increasing resistance to vancomycin and other glycopeptides in Staphylococcus aureus. Emerging Infectious Disease Journal 7, 327-332.

Vidhani, S., Mehndiratta, P.L. and Mathur, M. D. (2001). Study of methicillin resistant $S$. aureus (MRSA) isolates from high risk patients. Indian Journal of Medical Microbiology 29, 13-16.

Winn, W. Jr., Allen, S., Janda, W., Koneman, E., Procop, G., Schreckenberger, P. and Woods, G. (2006). Staphylococci and related Gram positive cocci. In: Winn, W. Jr., Allen, S., Janda, W., Koneman, E., Procop, G., Schreckenberger, P. and Woods, G. (eds.). Koneman's Color Atlas and Textbook of Diagnostic Microbiology. Lippincott Williams and Wilkins, USA. pp. 634-636.

Wootton, M., Howe, R. A., Hillman, R., Walsh, T. R., Bennett, P. M. and MacGowan, A. P. (2001). A modified population analysis (PAP) method to detect hetero-resistance to vancomycin in Staphylococcus aureus in UK hospital. Journal of Antimicrobial Chemotherapy 47, 399-403. 